\title{
Optofluidic circular grating distributed feedback dye laser
}

\author{
Yan Chen, ${ }^{\text {a) }}$ Zhenyu Li, M. David Henry, and Axel Scherer \\ Department of Electrical Engineering, California Institute of Technology, Pasadena, California 91125, USA
}

(Received 6 May 2009; accepted 25 June 2009; published online 22 July 2009)

\begin{abstract}
We demonstrate an optically pumped surface emitting optofluidic dye laser using a second-order circular grating distributed feedback resonator. We present a composite bilayer soft lithography technique specifically developed for the fabrication of our dye laser and investigate a hybrid polymer material system [poly(dimethylsiloxane)/perfluoropolyether] to construct high-resolution Bragg gratings. Our lasers emit single frequency light at low lasing thresholds of $6 \mu \mathrm{J} / \mathrm{mm}^{2}$. These optofluidic dye lasers can serve as low-cost and compact coherent light sources that are fully integrated within microfluidic analysis chips and provide an efficient approach to construct compact spectroscopy systems. (C) 2009 American Institute of Physics. [DOI: 10.1063/1.3186786]
\end{abstract}

The integration of optics with microfluidics, often referred to as optofluidics, has recently attracted great interest for its potential of building adaptive optical systems with many applications in biotechnology. ${ }^{1}$ In particular, considerable effort has been spent on constructing optofluidic dye lasers, as these enable the integration of coherent light sources with other microfluidic and optical functionalities. Dye lasers provide the opportunity of building more complete "lab-on-a-chip" systems, and various types of optofluidic dye lasers have been demonstrated in the past, including Fabry-Pérot cavities, ${ }^{2,3}$ ring resonators, ${ }^{4}$ and embedding distributed feedback (DFB) gratings. ${ }^{5-7}$ In particular, circular grating DFB laser resonators are considered suitable for integration in microfluidic devices because they combine low threshold lasing operation, surface emission, and compact output beam size. The circular laser cavity geometry also enables the large-scale integration of many laser arrays into compact devices, which can be very useful for many applications such as laser-induced fluorescence and spectroscopy. Many optofluidic devices demonstrated previously were constructed entirely of poly(dimethylsiloxane) (PDMS). However, the minimum feature sizes and the aspect ratios of geometries that can be replicated into PDMS-based optofluidic devices are limited by the low modulus and high surface energy of that polymer. ${ }^{8}$ In order to fabricate sub-200-nm grating structure with deep trenches, we find that it is necessary to employ an alternative and stiffer material, perfluoropolyether (PFPE), to form a laser resonator.

In this letter, we present a surface emitting optofluidic dye laser with a circular grating DFB structure defined into PFPE. However, it is still desirable to use PDMS for valving and pumping of liquids. This leads us to the general design of an optofluidic circular grating DFB dye laser, shown in Fig. 1(a), where the laser chip is a hybrid microfluidic device made of PDMS and PFPE. A microfluidic channel with embossed circular grating structures embedded is filled with organic dye solutions as the gain medium. The PDMS forms the microfluidic channel and serves as the upper cladding of the cavity, whereas the lower cladding material is PFPE, which forms the circular grating that provides the feedback necessary for the laser operation.

${ }^{a)}$ Electronic mail: yanc@caltech.edu.
The circular grating DFB geometry has been previously used to demonstrate nanoimprinted solid-state dye lasers ${ }^{9}$ to produce low threshold, single frequency lasing. This circular Bragg reflector structure provides a natural two dimensional extension of the basic DFB structure. The corrugations in a second-order grating provide both DFB and output coupling of the guided optical mode. ${ }^{10}$ Second-order gratings are often used to obtain surface emission since the first-order Bragg reflection from a second-order grating can couple radially propagating waves to vertically propagating plane waves, as illustrated in Fig. 1(b). The design parameters of our circular gratings fabricated are selected based on a combination of electromagnetic mode calculations and experimental results. In this letter, grating periods varying from 400 to $440 \mathrm{~nm}$ are chosen to match the second-order Bragg condition. We also use a $600 \mathrm{~nm}$ deep grating trench to compensate for the low refractive index contrast between the dye solution and the cladding.
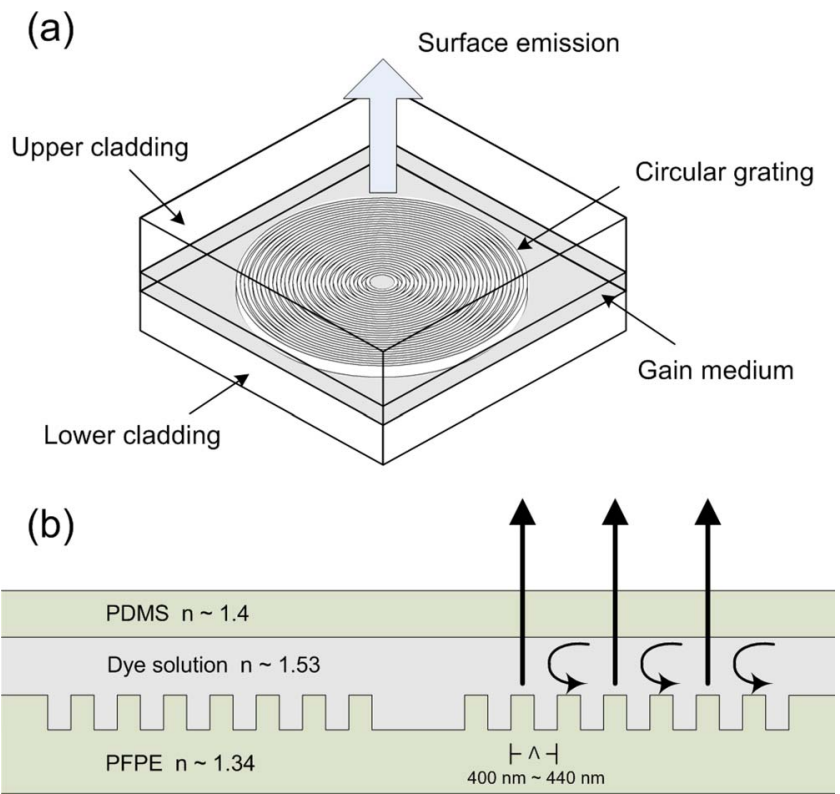

FIG. 1. (Color online) (a) General design of an optofluidic circular grating DFB dye laser cavity. (b) Illustration of the Bragg diffraction in the secondorder circular grating. First-order Bragg reflection induces coupling to vertical propagating waves, and second-order Bragg reflection leads to in-plane feedback. 
Photocurable PFPE has been reported by Rolland et al. as an alternative material for the construction of microfluidic devices ${ }^{11}$ and nanoimprint lithography. ${ }^{12}$ In this letter, we chose a commercially available form of PFPE (CN4000, Sartomer Co.) reported by Truong et al. ${ }^{13}$ With the higher Young's modulus of PFPE, we achieve replication of higher resolution and higher aspect ratio features than possible with PDMS. This geometric control gives rise to good optical confinement by the grating. Moreover, the low refractive index of PFPE $(n=1.341)$ makes it more suitable to serve as a cladding material for our laser device. However, reliable strong bonding between two layers of PFPE is very difficult. Therefore, our optofluidic dye laser is composed of two layers: a stiff layer of PFPE supported by a flexible layer of PDMS. This composite bilayer device structure ${ }^{14}$ combines the most attractive features of both materials, and it is very useful in defining functional microfluidic devices with nanoscale optical structures embedded.

To define our circular grating, silicon dioxide $\left(\mathrm{SiO}_{2}\right)$ was used as the mold material. In order to obtain a deep steepsided grating profile in $\mathrm{SiO}_{2}$, we used an inductively coupled plasma reactive ion etch (ICP-RIE) process, which required $\mathrm{Cr}$ as a highly selective etch mask. The mold was created by first thermally evaporating silicon dioxide with $\mathrm{Cr}$ before spin coating with electron beam resist [polymethylmethacrylate (PMMA)]. A circular grating pattern was then defined by electron beam lithography in a LEICA EBPG 5000+ e-beam writer with proximity correction capability. After mask transfer to the Cr underneath the exposed PMMA pattern by wet etching, the pattern was anisotropically transferred from $\mathrm{Cr}$ into the $\mathrm{SiO}_{2}$ substrate by using $\mathrm{C}_{4} \mathrm{~F}_{8}$ and $\mathrm{O}_{2}$ etch in an Oxford Instruments PlasmaLab System100 ICP-RIE 380.

The optofluidic dye laser design consists of two layers, a grating layer made of PFPE supported by a flexible PDMS backbone, and it is bonded to a thin layer of PDMS with flow channels inside. To prevent buckling of the wide PDMS flow channel, support pillars were fabricated within the flow channels. The fabrication of the hybrid material grating layer starts with aligning a thin PDMS layer on top of the grating mold to cover most of the mold except the grating areas. Then the PFPE is spin coated onto that mold, followed by UV exposure to cure the PFPE. After peeling off the thin PDMS layer, a mixture of PDMS prepolymer is poured onto the PFPE layer and thermally cured. This process forms a support layer for the composite PFPE/PDMS structure. The PFPE/PDMS layer is then peeled from the grating mold and bonded to another PDMS layer with the flow channel. Finally, the device is baked overnight to ensure bonding between the polymer layers. The bonding between PFPE and PDMS was found to be sufficient for laser chip operation. The fabrication process of the hybrid PFPE/PDMS laser chip is summarized schematically in Fig. 2.

Scanning electron microscopy (SEM) images of the $\mathrm{SiO}_{2}$ mold with a circular grating and its replica in PFPE are shown in Fig. 3. The image of PFPE was obtained using an environmental SEM (ESEM) in order to mitigate charging effects from the nonconductive polymer samples. From the ESEM image, we can see that the original geometry on the $\mathrm{SiO}_{2}$ mold is faithfully replicated in the PFPE with high resolution, and there is no observable lateral collapse between the grating lines even with aspect ratios above 3:1.

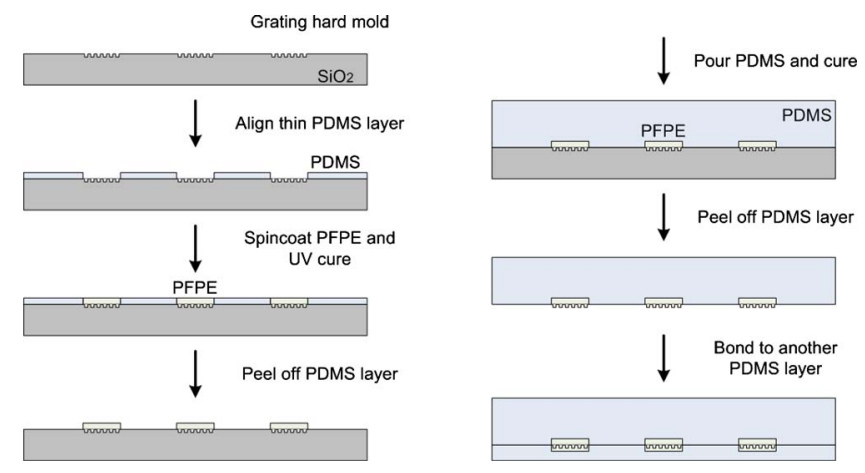

FIG. 2. (Color online) Schematic fabrication process of the hybrid PFPE/ PDMS laser chip.

The optofluidic dye laser chip was optically pumped with $532 \mathrm{~nm} 6 \mathrm{~ns} Q$-switched Nd:YAG laser pulses, focused through a $20 \times$ lens above the chip. A $10 \times$ microscope objective was used to collect the light emission from the bottom side of the chip. The emission spectra were acquired using an Ocean Optics USB2000 spectrometer. A pressure source was used to circulate the dye solution in the flow channel through the laser cavities. We used rhodamine $6 \mathrm{G}$ (exciton) laser dye, chosen to match its absorption peak with the cavity resonator frequency, and a mixture of benzyl alcohol and methanol with refractive index of 1.53 was used as the high index solvent.

A typical single frequency laser spectrum, measured from one of the circular grating dye lasers, is shown in Fig. 4. The lasing wavelength for this device is $581.13 \mathrm{~nm}$, and lasing occurs near the Bragg resonance, determined by the Bragg equation $m \lambda_{\text {Bragg }}=2 n_{\text {eff }} \Lambda$, where $m=2$ is the order of diffraction, $n_{\text {eff }}$ is the effective refractive index of the propagation mode, and $\Lambda$ is the grating period. In this laser, with the grating period of $410 \mathrm{~nm}$, the $n_{\text {eff }}$ is 1.42 , which is close to the effective index simulation result of the waveguide. The laser is azimuthally polarized, ${ }^{15}$ and only the fundamental mode exhibits here because it overlaps most efficiently with the gain spectrum of rhodamine. The inset shows the variation in the output laser power as a function of pump energy. The threshold pump fluence is estimated to be $6 \mu \mathrm{J} / \mathrm{mm}^{2}$ for this device. This relatively low pump threshold is within
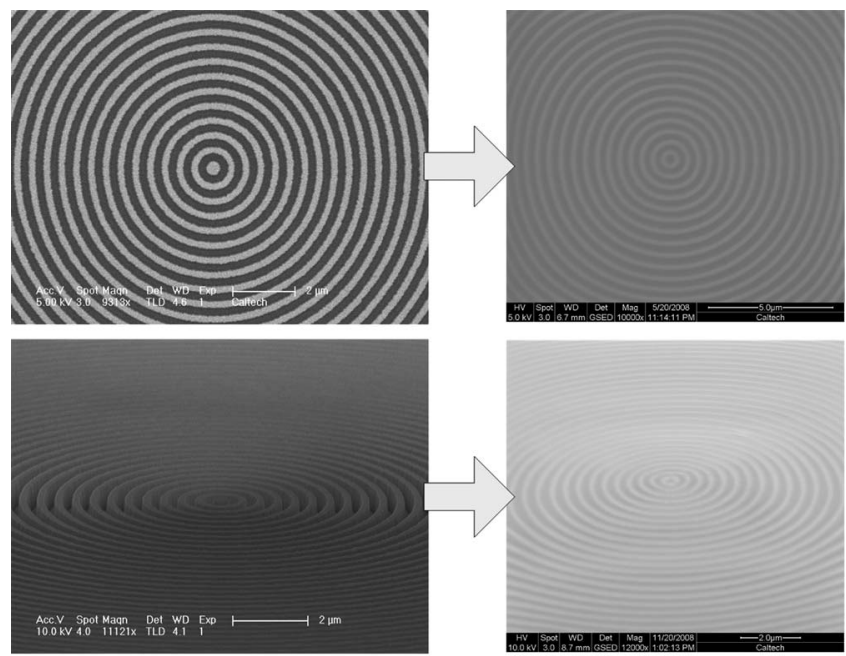

(a)

(b)

FIG. 3. (a) SEM images of $\mathrm{SiO}_{2}$ mold. (b) ESEM images of PFPE replica. 


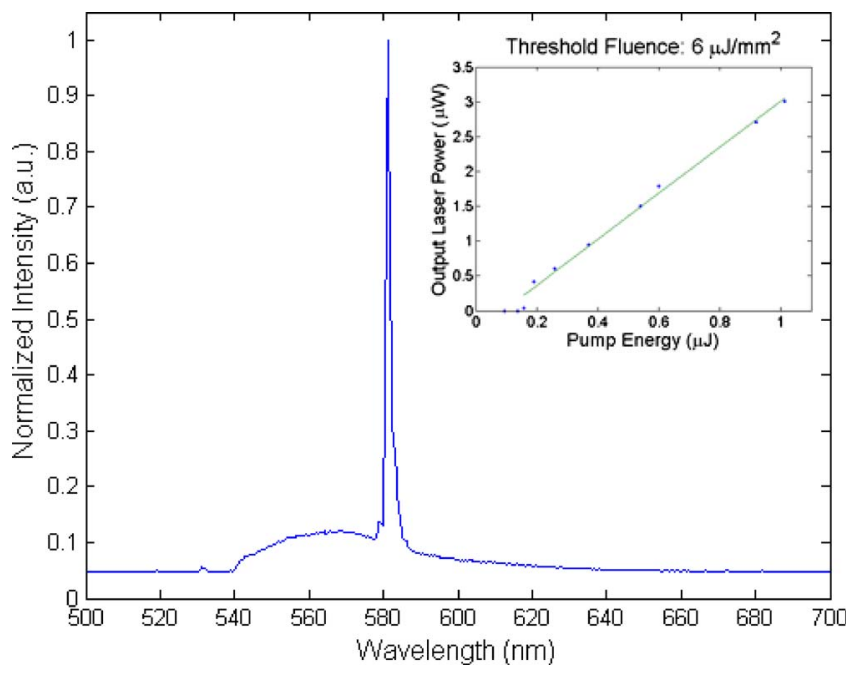

FIG. 4. (Color online) Optofluidic circular grating DFB dye laser spectrum. Inset: the output laser power vs pump energy curve. The threshold pump fluence is $6 \mu \mathrm{J} / \mathrm{mm}^{2}$.

the reach of high power laser diodes operating in pulsed mode. In order to realize light-emitting diode pumped optofluidic dye lasers, however, further reduction in the dye laser threshold is required. The small size and surface emitting geometry of the laser cavity also provide a simple but efficient scheme to construct laser arrays with geometrically controlled emission wavelengths, pumped with a single wavelength source.

We also observed a decrease in the laser emission intensity with increasing pump exposure time during laser operation, presumably a result of bleaching of the dye molecules. Circulating the dye solution with a higher flow rate would prevent the dye molecules from photobleaching under pulsed operation. One of the attractive features of an optofluidic circular grating dye laser is that the dye is constantly changed, thereby increasing the device lifetime. Integration of microfluidic circuits on the laser chip also enables wavelength tuning by simply mixing dye solutions with different refractive indices. The laser dye we used in this letter has a lasing wavelength range from 563 to $597 \mathrm{~nm}$; other laser dyes can also be used to expand the output wavelength range with the solvent indices varying from 1.4 to 1.54 .
In summary, we have demonstrated a second-order circular grating DFB laser embedded in an optofluidic dye delivery system. We obtain surface emitting single frequency lasing at a relatively low pumping threshold. To construct these circular grating dye lasers, we have developed a hybrid PFPE/PDMS soft lithography technique that enables the fabrication of low-cost, disposable, and high quality dye laser arrays. This polymer heterostructure also provides a convenient method for fabricating optofluidic device with nanoscale optical structures embedded within traditional PDMS flow channels.

This research was supported by the NSF under the Center for Materials and Devices for Information Technology Research (MDITR) and the Center for Integrated Access Networks (CIAN). David Henry gratefully acknowledges funding by the John and Fannie Hertz Foundation.

${ }^{1}$ D. Psaltis, S. R. Quake, and C. H. Yang, Nature (London) 442, 381 (2006).

${ }^{2}$ J. C. Galas, J. Torres, M. Belotti, Q. Kou, and Y. Chen, Appl. Phys. Lett. 86, 264101 (2005).

${ }^{3}$ D. V. Vezenov, B. T. Mayers, R. S. Conroy, G. M. Whitesides, P. T. Snee, Y. Chan, D. G. Nocera, and M. G. Bawendi, J. Am. Chem. Soc. 127, 8952 (2005).

${ }^{4}$ S. I. Shopova, H. Y. Zhou, X. D. Fan, and P. Zhang, Appl. Phys. Lett. 90, 221101 (2007).

${ }^{5}$ S. Balslev and A. Kristensen, Opt. Express 13, 344 (2005).

${ }^{6}$ Z. Y. Li, Z. Y. Zhang, T. Emery, A. Scherer, and D. Psaltis, Opt. Express 14, 696 (2006).

${ }^{7}$ M. Gersborg-Hansen and A. Kristensen, Appl. Phys. Lett. 89, 103518 (2006).

${ }^{8}$ H. Schmid and B. Michel, Macromolecules 33, 3042 (2000).

${ }^{9}$ Y. Chen, Z. Li, Z. Zhang, D. Psaltis, and A. Scherer, Appl. Phys. Lett. 91, 051109 (2007).

${ }^{10}$ G. A. Turnbull, A. Carleton, G. F. Barlow, A. Tahraouhi, T. F. Krauss, K. A. Shore, and I. D. W. Samuel, J. Appl. Phys. 98, 023105 (2005).

${ }^{11}$ J. P. Rolland, R. M. Van Dam, D. A. Schorzman, S. R. Quake, and J. M. DeSimone, J. Am. Chem. Soc. 126, 2322 (2004).

${ }^{12}$ J. P. Rolland, E. C. Hagberg, G. M. Denison, K. R. Carter, and J. M. De Simone, Angew. Chem. Int. Ed. 43, 5796 (2004).

${ }^{13}$ T. T. Truong, R. S. Lin, S. Jeon, H. H. Lee, J. Maria, A. Gaur, F. Hua, I. Meinel, and J. A. Rogers, Langmuir 23, 2898 (2007).

${ }^{14}$ T. W. Odom, J. C. Love, D. B. Wolfe, K. E. Paul, and G. M. Whitesides, Langmuir 18, 5314 (2002).

${ }^{15}$ R. H. Jordan, D. G. Hall, O. King, G. Wicks, and S. Rishton, J. Opt. Soc. Am. B 14, 449 (1997). 